Voix et Images

\title{
Le personnage français dans quelques romans québécois contemporains
}

Neil B. Bishop

Volume 13, numéro 1 (37), automne 1987

Suzanne Lamy

URI : https://id.erudit.org/iderudit/200687ar

DOI : https://doi.org/10.7202/200687ar

Aller au sommaire du numéro

Éditeur(s)

Université du Québec à Montréal

ISSN

0318-9201 (imprimé)

1705-933X (numérique)

Découvrir la revue

Citer cet article

Bishop, N. B. (1987). Le personnage français dans quelques romans québécois contemporains. Voix et Images, 13(1), 82-103. https://doi.org/10.7202/200687ar d'utilisation que vous pouvez consulter en ligne.

https://apropos.erudit.org/fr/usagers/politique-dutilisation/ 


\title{
Le personnage français dans quelques romans québécois contemporains 1
}

\author{
par Neil B. Bishop, Université Memorial de Terre-Neuve
}

\section{À la mémoire de Louis Marie Perrot}

Peu de travaux touchent à notre sujet précis; des études intéressantes existent sur des sujets apparentés: A. Vanasse a consacré une série d'articles à l'étranger au sens de l'inconnu, le «survenant» chez Guèvremont, par exemple; plus près de notre sujet, A. Sirois a étudié «l'Étranger de race et d'ethnie dans le roman québécois», W. de Roussan, le personnage de l'étrangère, B.-Z. Shek, V. Teboul, et A.-V. Abouteboul le personnage juif, et R. Hathorn les personnages anglophones 2 . Ces travaux évoquent fort utilement la représentation du type de personnage étudié, mais définissent peu leurs assises théoriques, et tiennent peu compte de la dimension actantielle. Leur utilisation pour l'étude du personnage français est limitée. Celui-ci se distingue en effet fondamentalement de personnages d'autres appartenances, et cette remarque de Claude Galarneau sur les représentations québécoises de la France s'applique aussi aux Français fictifs du roman québécois:

Dans le cas de la France vue par les Français canadiens, il ne s'agit pas du même type de représentation que celle des Français qui voient l'Allemagne, l'Angleterre, l'Italie ou les États-Unis, mais bien d'une collectivité issue de l'autre par filiation directe $[. . .]^{3}$

Notre but principal est d'étudier le phénomène textuel concret évoqué dans notre titre; mais il convient d'indiquer brièvement nos présupposés théoriques. Il faut reconnaître que la notion de personnage romanesque ne va plus de soi. Notion «périmée» pour Robbe-Grillet ${ }^{4}$ et d'autres tenants du «Nouveau Roman», le personnage ne fait point l'objet d'une entrée dans le Gradus de Bernard Dupriez. Greimas et Courtès remplacent la notion de personnage par celles d'actant et

1 Cet article, extrait d'un projet plus vaste portant sur la France et le personnage français dans le roman québécois, est une version remaniée d'une communication présentée à Winnipeg le 29 mai 1986 lors du colloque de l'A.L.C.Q.

2 On trouvera une bibliographie détaillée de ces travaux en fin d'article.

3 Claude Galarneau, «la Légende napoléonienne au Québec», dans F. Dumont et Y. Martin (dir.) Imaginaire social et représentations collectives, p. 163.

4 Alain Robbe-Grillet, Pour un nouveau roman, Paris, Minuit, 1963, p. 25-28. 
d'acteur 5 . Or, l'actant et l'acteur sont pour nous deux dimensions d'un même phénomène textuel et le terme «personnage» désigne ce phénomène textuel dans son ensemble. Le volet «acteur» semble quant à lui comporter deux aspects, comme l'a indiqué Philippe Hamon qui voit dans le personnage un composé de trois aspects: un aspect «actant», un aspect «support-signifiant», et un aspect «signifié», ces deux demiers constituant l'acteur6.

À l'aide de Greimas, de Courtès et de Hamon, nous avons ainsi établi une définition opératoire du personnage romanesque: un signe textuel à deux composantes principales, l'actant et l'acteur, cette dernière comportant une dimension «support-signifiant» et une dimension «signifié». La dimension «support-signifiant» de l'acteur comprend tout aspect non actantiel du personnage: apparence physique, psychologie, etc. (ces traits peuvent influencer et même déterminer ses actes et donc sa fonction actantielle). Le fonctionnement signifiant de chacune des deux composantes principales, l'actant et l'acteur, est intimement lié à celui de l'autre, car ils interagissent pour produire la signification du personnage. Celui-ci, en tant quactant, constitue une «force orienté» (Souriau7): dans notre corpus le personnage français se révélera une force orientée tantôt favorablement, tantôt défavorablement par rapport à un ou des protagoniste(s) québécois en quête de bonheur et d'épanouissement. Cette fonction actantielle du personnage français sera le plus souvent le facteur principal déterminant son statut positif ou négatif, d'adversaire ou d'adjuvant. Mais la composante actorielle conservera une réelle importance: le personnage, en tant que support signifiant, comportera des traits signifiants dont les signifiés apporteront au lecteur un complément d'information; dans le cas d'un personnage français important, celui de Marie Jourdan dans le Crime d'Ovide Plouffe, la dimension actorielle est au moins aussi importante que sa dimension actantielle dans la détermination de sa fonction signifiante.

S'il importe de tenir compte de ces diverses composantes du personnage, une étude appliquée tendra à procéder par glissements constants de l'une à l'autre; car, tout composite qu'il soit, le personnage romanesque constitue une entité dont les diverses composantes interagissent et s'influencent mutuellement, en un va-etvient incessant que doit souvent épouser l'analyse.

Marc Angenot, dans son Glossaire pratique de la critique contemporaine, confirme que la notion de personnage n'est [...] pas éliminée de la sémiotique narrative et définit le personnage comme le point de convergence d'un certain

5 A.J. Greimas et J. Courtès, Sémiotique, dictionnaire raisonné de la théorie du langage, Paris, Hachette, 1979, p. 274.

6 Philippe Hamon, «Pour un statut sémiologique du personnage», Littérature, no 6, mai 1972 , p. 104.

7 Etienne Souriau, les Deux cent mille situations dramatiques, Paris, Flammarion, 1950. 
nombre de traits isotopes, d'indices susceptibles de se constituer en système ${ }^{8}$. Cette définition complète utilement la précédente. Pertinente aussi est l'affirmation, par des auteurs comme Hamon, Paquin et Reny, selon laquelle le personnage romanesque n'est pas forcément un être humain fictif, mais peut être un objet ou une abstraction9. Dans notre corpus, la France et surtout la langue et la culture française, métonymiquement liées au personnage français humain, en constituent des aspects souvent indissociables, et forment avec lui un ensemble que nous appellerons parfois le «personnage français complexe» pour souligner qu'alors il convient d'accorder une attention particulière au pays, à la langue ou à la culture du personnage 10 .

Quant à notre corpus, il sera nécessairement limité, dans le cadre de cette étude. L'établissement du corpus pose d'autres problèmes méthodologiques. Fautil inclure des romans tels que Héloïse, d'Anne Hébert, dont l'action se situe en France et dont les personnages sont français? Les Chambres de bois pose en fait le même problème. Pour Anne Hébert, le pays de la première partie de ce roman, (la critique l'a longtemps rattaché au pays de l'amiante québécois) lui a été inspiré plutôt par le nord de la France tel qu'elle l'a vu en revenant vers Paris lors d'un voyage en Belgique. Or, rien dans ces romans ne suggère que l'appartenance française des personnages, sur laquelle ces récits n'insistent guère, influe sur leur fonction actantielle ou leur qualification actorielle 11 . Par contre, le Matou de Yves Beauchemin désigne souvent Aurélien Picquot comme le Français; la

8 Marc Angenot, Glossaire pratique de la critique contemporaine, Montréal, Hurtubise HMH, 1979, entrée «Personnage».

9 Voir Hamon, op. cit., p. 87-8; M. Paquin et R. Reny, la Lecture du roman, Mont-Saint-Hilaire, Qué, La Lignée, 1975, p. 67.

10 Jacques Allard, dans ses commentaires suite à notre communication, a confirmé que lui aussi voit comme étant intimement liés, dans le roman québécois, les représentations de personnages français et celle d'autres aspects de la civilisation française. Il a suggéré qu'un aspect pertinent de la problématique serait l'attitude manifestée, dans et par les romans québécois, envers les formes narratives héritées de la littérature française, ou encore envers le français comme langue narrative. Il s'agit là, nous semble-t-il, de deux problèmes différents, importants tous deux. Ces perspectives sont passionnantes, encore que leur étude dépasserait le cadre du présent travail (nous espérons les intégrer dans le projet plus vaste évoqué au début de cet article). Notre étude ci-dessous de Maryse de Francine Noël évoque le problème de l'attitude envers les diverses variantes du français. Nous remercions Monsieur Allard de ses utiles remarques.

11 En outre, un roman fait par un auteur québécois mais dont tous les personnages, ainsi que le cadre, sont français, pose de tout autres problèmes que ceux posés par des personnages français évoluant parmi des personnages québécois. Dans le premier cas, on est obligé, par exemple, de se poser la question de savoir dans quelle mesure le cadre et les personnages étrangers (français ou autre) sont symboliques du Québec et des Québécois, et dans quelle mesure ils restent étrangers. Cette problématique extrêmement complexe dépasse le cadre du présent travail; nous espérons l'aborder ailleurs. 
Duchesse et le roturier de Michel Tremblay met en texte des personnages non seulement identifiés comme français, mais «référentiels», selon le terme de Hamon 12, puisqu'ils correspondent à des personnes réelles, célèbres, symboliques de la France - Valéry Giscard d'Estaing et Tino Rossi; le Crime d'Ovide Plouffe évoque Charles Trenet, Maurice Chevalier, Aznavour, parmi d'autres, et comporte des personnages français des plus importants; l'amant de Marie-Lyre dans Maryse de Francine Noèl est précisément identifié comme un Français (comme le sont d'autres personnages de ce roman) et c'est largement son appartenance française qui entraine des conséquences pour leur relation; MamanParis Maman-la-France de Claude Jasmin situe son action explicitement en France et thematise fortement l'appartenance française de bon nombre de ses personnages ${ }^{13}$. Pour être pertinent à une étude du personnage français, il faudra sans doute que le roman comporte des personnages dont l'appartenance française influe de façon significative sur leur comportement et leur signification: le plus souvent, le personnage sera explicitement et fortement désigné comme français et posé dans un rapport différentiel avec des personnages non français - le plus souvent québécois.

Notre corpus consistera donc en ces cinq romans, qui seront abordés en deux mouvements. Nous examinerons, dans un premier temps, les personnages français (d'abord les opposants, ensuite les adjuvants) dans Maryse, la Duchesse et le roturier, le Matou et Maman-Paris Maman-la-France. Dans un deuxième temps, nous étudierons le Crime d'Ovide Plouffe, roman traité à part en raison de la complexité considérable qu'y revêt la problématique du personnage français. Il offre de plus l'intérêt particulier de donner un versant spécifiquement littéraire à cette problématique. Ce corpus de cinq romans est vaste de ses quelques deux mille deux cents pages; et restreint par rapport au corpus possible: le caractère peu étudié du sujet oblige en effet à une analyse fouillée et rigoureuse du personnage français dans un corpus limité. Les cinq romans permettront néanmoins de constater une gamme des fonctions possibles du personnage français par rapport aux protagonistes québécois, depuis un cas limite de la fonction d'opposant (le Crime d'Ovide Plouffe) à un cas limite de la fonction d'adjuvant (Maman-Paris Maman-la-France), en passant par divers degrés ou de mélanges d'adjuvance et d'opposition. Cette gamme des fonctions possibles fera ressortir

12 Ph. Hamon, op. cit., p. 95.

13 Notre corpus, une portion seulement du corpus possible, et présenté à simple titre d'échantillon en raison de l'espace limité dont nous disposons, consistera en cinq romans récents: Yves Beauchemin, le Matou, Montréal, Québec/ Amérique, 1981, 583 p.; Claude Jasmin, Maman-Paris Maman-la-France, Montréal, Leméac. 1982, 344 p.; Roger Lemelin, le Crime d'Ovlde Plouffe, Québec, ETR, 1982, 500 p; Francine Noĕl, Maryse, Montréal, VLB Éditeur, 1983, 426 p.; Michel Tremblay, la Duchesse et le roturier, Montréal, Leméac, $1982,387 \mathrm{p}$. Toute référence à ces ouvrages se fera dans notre texte par le numéro de page précédé des letrres suivantes: Mat. (le Matou), MPMF (Maman-Parls Maman-la-France), COP (le Crime d'Ovide Plouffe) Mar. (Maryse), DR (la Duchesse et le roturier). 
les points précis, les valeurs en fonction desquelles l'adjuvance ou l'opposition du personnage français est perçue comme particulièrement pertinente par les protagonistes québécois.

Commençons par le personnage français comme opposant. Dans Maryse, de Francine Noël, le professeur français André Breton humilie sa maîtresse québécoise Marie-Lyre et devient ainsi opposant par rapport à sa quête de bonheur et de dignité comme femme et Québécoise. Or, le fait d'être français est lié à la fonction d'opposant chez Breton: il mortifie Marie-Lyre pour avoir mis le mot espèce au masculin; et quand elle lui dit qu'il [est] le chum le plus fin qu' [elle] ai[t] jamais eu! Breton répond que le mot chum est laid et vulgaire (Mar., p. 107). Son statut d'opposant vient donc de ce qu'il est français et nie à MarieLyre le droit à sa spécificité langagière québécoise; la lutte se situe au niveau du droit à l'expression langagière libre et authentique. Ce personnage français s'arroge le pouvoir de décider de la langue française; le personnage québécois conteste ce monopole. Selon Maryse, cette relation d'opposition caractérise tout rapport entre Français et Québécois: C'est toujours pareil avec les Français, [...] quand on leur parle, on a l'impression de passer un examen (Mar., p. 107). L'instance narrative elle-même marque le professeur Breton d'un coefficient négatif en affirmant avec ironie qu'il répétait depuis dix ans les mêmes sublimes cours sur le vers anapestique blanc (Mar., p. 106); ce qui renforce son statut d'opposant au peuple québécois à qui, suggère le livre, il n'en donne pas assez pour ses impôts.

Un autre professeur français, Léon Bardanne, est présenté de façon négative -dans Maryse, car il est le tenant hyper-jargonisant d'une théorie littéraire alliant la méthode chomskienne et celle du carbone 14! (Mar., p. 81) Il est un de ces littérologues que Maryse vit comme des opposants dans sa quête de bonheur et d'affirmation de soi. À nouveau, le caractère d'opposant du personnage français est lié à l'emploi du langage comme instrument de pouvoir ${ }^{14}$.

La problématique entre personnages français et québécois se réduit à son seul aspect langagier dans la lutte qui oppose Maryse au génie de la langue française. Ce lutin critique le langage québécois de l'héroïne, cherche à lui imposer la norme française, et tend à paralyser ses tentatives d'expression jusqu'à ce qu'elle réussisse à le noyer dans une bouteille d'encre, événement qui signale l'intensité de l'opposition entre ces deux personnages.

14 Le cas de Léon Bardanne illustre toutefois la complexité de notre problématique qui entre ici en interaction avec une problématique féministe. Bardanne, ridiculisé par l'instance narrative qui lui attribue un jargon, une théorie et une méthode des plus fantaisistes, est applaudi par les étudiants, par les gars surtout, ces étudiants masculins, tels que Michel, en la majorité desquels Maryse finira par voir des opposants le plus souvent par rapport à sa quête du bonheur. Maryse ne s'intéresse nullement au cours de Bardanne (Mar., p. 81). 
Le personnage français et sa langue font partie ici d'un ensemble plus vaste qui comprend la culture française. Ses rapports conflictuels avec le personnage québécois se révèlent clairement quand Maryse pense à l'attirance qu'exerce sur elle un personnage de culture espagnole. Celle-ci offre l'avantage d'être une culture n'offrant que de plaisantes différences avec la nôtre et peu de points de comparaison. On se comparait trop aux Français, on les prenait pour modèles et on avait tort: ça finissait toujours mal, on en venait d̀ se haïr (Mar., p. 261).

La Duchesse et le roturier de Michel Tremblay met en scène deux personnages français référentiels: Tino Rossi et Valéry Giscard d'Estaing. Giscard d'Estaing, professeur au collège Stanislas, a beau trouver plutôt charmants les Canadiens français (DR, p. 92), ceux-ci le vivent comme force négative: Maintenant qu'un Français était parmi eux, personne n'osait plus parler [...] Seule Mercedes osa murmurer une phrase, qui résumait d'ailleurs la pensée de tout le monde: «Y en faut rien qu'un pour toutes nous clouer le bec, hein?» (DR, p. 82). Tout comme dans Maryse, le problème du rapport entre personnages français et québécois est largement structuré par la différence langagière. Dès que Giscard ouvre la bouche, les Québécois le savent français et le voient comme opposant. En outre, un fossé d'incommunicabilité le sépare des Québécois, ce qui renforce son statut d'opposant en frustrant ses interlocuteurs québécois. Ainsi, dans une scène, et comique et triste, la Poune, flattée du compliment de Giscard, lui demande son nom pour pouvoir l'ajouter à ses trophées de chasse - c'est-àdire, faire progresser sa quête du bonheur en se valorisant. Le personnage répond:

Valéry Giscard d'Estaing. Il avait laissé tomber le nom comme on aurait autrefois déposé une carte de visite dans un plateau d'argent.

La Poune fronça les sourcils et éleva la voix: Je vous ai pas demandé le nom de votre fille, j'vous ai demandé vot'nom à vous! Furieuse, elle rentra dans la loge. Valérie! Y veut-tu rire de nous autres, lui? (DR, p. 85)

Ce sentiment d'être moqué pour son langage par le Français est présent chez Marie-Lyre dans Maryse; il a durement marqué Gérard Bleau, dans la Duchesse, lorsqu'il participait à la libération de la France lors de la Deuxième Guerre mondiale (DR, p. 102). Ce chauffeur de tramway introduit de nouvelles données à la problématique des rapports entre personnages français et québécois. D'une part, il précise que ces moqueries n'ont pas eu lieu en Normandie, oul le monde parlait un peu comme nous autres, mais à Paris, où on faisait rire de nous autres, rare! Aussitôt qu'on ouvrait la bouche tout le monde se roulait à terre! (DR, p. 102). Bleau affirme ainsi une différence démarquant les rapports entre Québécois et Français provinciaux de ceux existant entre Québécois et Parisiens. D'autre part, Bleau continue ainsi: Savez-vous ça, vous, qu'on le savait pas qu'on avait un accent avant de se le faire dire bête de même! Moé, avant tout ça, j'tais sûr que c'tait vous autres qui aviez un accent! (DR, p. 102). Bleau annonce ainsi la valorisation de l'accent québécois par l'instance narrative de Maryse qui, parlant 
d'un personnage d'origine espagnole, déclare qu'il avait rapidement appris notre français qu'il parlait sans aucun accent, c'est-d̀-dire avec le nôtre (Mar., p. 256).

Cette valorisation du langage québécois est présente dans la lutte de Maryse et de Marie-Lyre contre les représentants d'une norme langagière française: MarieLyre deffend avec vigueur et brio son emploi du mot chum au sens québécois (Mar., p. 107-109); il n'en reste pas moins qu'il s'agit là d'un terme emprunté à l'anglais, et Maryse a parfois recours au québécois, mais souvent à l'angloaméricain comme arme contre le génie de la langue française (il est vrai qu'elle est anglophone par son père irlandais, francophone par sa mère québécoise). La valorisation du français québécois n'est donc pas le seul enjeu de la lutte de Marie-Lyre et de Maryse contre la norme française: elle leur permet aussi d'affirmer leur indépendance vis-à-vis d'une instance d'autorité française. Cette affirmation se fait à l'aide de l'anglais, synecdoque et symbole de l'américanité de ces personnages qui l'utilisent pour contrer les pressions excessives de l'héritage ancestral français; une fois que Maryse aura noyé le génie de la langue française, elle décidera d'elle-même d'écrire en un français standard (Mar., p. 367). Sa décision sera alors personnelle, indépendante et adulte.

Le Matou comporte un personnage français féminin, Mademoiselle Lydie, assistante d'une femme d'affaires québécoise. Florent, le héros québécois du roman et son épouse Élise voient Lydie comme une force hostile, opposée à eux. Parmi les composantes de Lydie en tant que support-signifiant, plusieurs relèvent sans doute d'un stéréotype de la Française d'un certain âge d'origine petitebourgeoise ou bourgeoise. Sa phrase, comme une mince feuille de glace (Mat., p. 271) sa tête haute, sa démarche précieuse (Mat., p. 267), aboutissent à son expression de dédain poli (Mat., p. 272), à sa lèvre dédaigneuse (Mat., p. 315). Ce comportement a sans doute pour signifié l'appartenance française bourgeoisante, celle qui dans notre corpus semble liée le plus indissolublement à la fonction d'opposant.

Quant au personnage français adjuvant, il est le cas le plus récurrent de notre corpus.

Marie-Lyre, à qui le roman Maryse inflige l'amant français que l'on sait, passe néanmoins un séjour à Paris afin de parfaire sa formation d'actrice. À son retour, la narration présente d'abord une vision négative de la mère patrie:

Marie-Lyre revint [...]. Peu contaminée par la culture, les tics et les modes de l'ancienne mère patrie, elle reprit facilement le rythme de la vie montréalaise, évitant ainsi à son entourage de subir les retombées de deux années de snobisme parisien. On la jugea intacte. "T'as pas changé, MLF!» disait-on. (Mar., p. 281)

Subitement, le récit se démarque de cette vision de l'ancienne mère patrie, suggérant ainsi que cette vision n'était que celle des amis montréalais de MarieLyre. Quand on lui disait: T'as pas changé!, Marie-Lyre 
trouvait ça un peu déprimant, mais souriait: elle savait bien qu'elle avait changé, ayant bien décidé d'être heureuse maintenant, et bien dans sa peau, quoi qu'il lui arrive ou ne lui arrive pas. Elle y parvenait pendant de longues heures. (Mar., p. 281)

Cette conquête de la sagesse et du bonheur semble avoir eu lieu grâce au contact avec la civilisation française et avec les Français qui revêtent ainsi le statut d'adjuvants de Marie-Lyre dans sa quête du bonheur.

Maryse elle-même, malgré ses démêlés avec le génie de la langue française, en vient à voir dans cette langue un adjuvant dans sa quête du bonheur. À propos de ses écrits futurs, Maryse dit: Je vais me faire plaisir. Je vais rédiger tout ça dans une langue châtiée, impeccable. Jusse pour le fun! (Mar., p. 367).

Dans la Duchesse, Tino Rossi fonctionne comme adjuvant par rapport à la quête québécoise du bonheur et de la fierté. Dès avant l'annonce de son spectacle, ses chansons sont connues et appréciées de bon nombre des personnages québécois; une fois annoncé le spectacle, ceux-ci sont remplis d'une anticipation fébrile mais joyeuse. Le récit présente un ténorino sympathique, et ses effets sur les Québécois sont positifs. Assister au spectacle de Rossi procure aux spectateurs québécois un moment de bonheur et les valorise dans leur milieu. Le Québécois Édouard s'habille publiquement en femme pour la première fois de sa vie, exprimant enfin ouvertement sa vérité intérieure. Il s'agit pour lui d'un véritable moment de libération. La différence entre les effets des deux personnages français référentiels, Giscard d'Estaing et Tino Rossi, sur les personnages québécois résulte surtout du type de culture et de classe sociale que chacun représente. Giscard (comme Mademoiselle Lydie) incarnerait le Français bourgeois, avec les connotations stéréotypées de morgue et de sentiment de supériorité que cela peut comporter; Rossi, chanteur populaire, ne fait pas peur aux Québécois populaires qui au contraire l'adorent, tout comme l'élite ${ }^{15}$.

L'emploi par Tremblay de personnages référentiels français, vedettes de la politique et de la chanson, peut également viser le succès commercial du roman en France. Le personnage français, même opposant du personnage québécois, peut se muer en adjuvant de l'auteur québécois dans sa quête d'une réussite sur le marché français. De telles considérations pourraient ne pas être étrangères à la place importante que l'auteur du Matou accorde à Aurélien Picquot.

15 Il arrive dans la Duchesse et le roturier que la grande culture française soit perçue comme adjuvant par les personnages québécois dont l'un dira $j$ 'lis Balzac pour m'aider à vivre! (DR, p. 2122) Ce roman tendra ultérieurement à substituer a cette utilisation de la culture française une prise de conscience approfondie de la réalité québécoise à l'aide d'une culture québécoise présentée comme adjuvant plus valable des personnages québécois, culture qui prendra la forme du Bonheur d'occasion de Gabrielle Roy. 
Le personnage français d'Aurélien Picquot est surtout adjuvant pour le couple central et québécois, Élise et Florent. Tout au long de leur lutte contre Egon Ratablavasky, Picquot les aide au maximum par un soutien moral et matériel, ainsi que par un engagement qui le conduit à la violence et à deux hospitalisations (Mat., p. 191-192; p. 581).

Comme à Lydie, le Matou attribue à Picquot nombre de traits stéréotypiques du Français, mais ce ne sont plus les mêmes. Physiquement, il y a ses longues moustaches noires et pointues dûment tortillées. C'est un excellent chef de cuisine. Il est anticlérical (Mat., p. 144) et même athée (Mat., p. 555), rationaliste - «Je sais ce dont vous avez besoin: une bonne cure de rationalisme. Diderot, Voltaire, Renan, voila les gens qu'il vous faut fréquenter!» dit-il à Florent (Mat., p. 572) - voire cartésien; mais aussi d'une grande bonté, d'une sensibilité romantique, d'une émotivité volcanique. Picquot aime évoquer ses exploits lors de la guerre - prétendant même avoir servi avec le lieutenant Alexis de Bellevoie, un ami intime du général de Gaulle [...] dans une mer infestée de sous-marins allemands (Mat., p. 556). Il a un net penchant pour les femmes et le cognac, et des sentiments peu amènes pour les Anglais et les Américains (qu'il appelle ignobles Amerloques, p. 46, cochons qui sont en train de saper notre civilisation, p. 516; notons le notre qui témoigne sans doute d'un sentiment de communauté culturelle avec ses interlocuteurs québécois). Surtout, Picquot attache une grande importance à la qualité, en matière de cuisine d'abord; dans tous les autres domaines de la vie ensuite (d'où, en partie, son antiaméricanisme, voir p. 46, 71, 73, 365-666, 516). Il s'agit d'un esthétisme polymorphe. Or, la quasi-totalité de cette liste de traits rattachés par le roman au support-signifiant Aurélien Picquot renvoient à un seul et même signifié: le Français. Picquot est très fortement codé comme français, appartenance que le roman signale de multiples façons, comme pour signifier que c'est là l'aspect le plus important de ce personnage - avec son rôle d'adjuvant parfait du héros québécois.

Picquot ne pose aux personnages québécois aucun problème de langage. C'est grâce à lui que sont créśs ces symboles - à l'instar de Picquot lui-même — de la solidarité franco-québécoise que sont les grands-mères, vieille recette savoyarde que ressuscite Picquot et que le restaurant fera passer pour une ancienne recette québécoise (Mat., p. 85-86). Picquot déclare avoir abandonné la haute cuisine française pour une branche plus basse, mais solide, la cuisine québécoise (Mat., p. 68). La cuisine de Chez Florent témoigne fortement de la solidarité de Picquot avec les Québécois, voire de la solidarité entre les deux cultures, française et québécoise, représentées métonymiquement par leurs cultures culinaires:

Le menu s'appuyait pour l'essentiel sur la cuisine traditionnelle québécoise, [...] discrètement affinée, complétée [...] par quelques spécialités françaises [...] Mais comme l'on s'adressait d̀ une clientèle populaire facile à effaroucher, Élise avait suggéré de rebaptiser les plats européens pour leur donner un petit air de chez nous. La crème de 
brocoli, la crème de carottes, le potage Parmentier et le velouté Aurore se retrouvèrent sous la rubrique des BONNES SOUPES DE MA TANTE DÉLIMA avec la soupe aux pois et la soupe aux choux. Le coq au vin se présentait comme un kfricot de poulet à la mode du Bas du Fleuve». Le sauté de veau [...] portait le nom de "fricassée de veau de Saint-Félicien». [...] La section des desserts affirmait clairement sa double allégeance québécoise et française [...] (Mat., p. 515-516, nous soulignons)

La solidarité franco-québécoise est ici tellement forte qu'elle se rapproche du fusionnel et de l'identité. Cette double allégeance québécoise et française est l'un des signifiés fondamentaux du Matou, comme l'indique le groupe central de personnages composé de Picquot, Florent, Élise et monsieur Émile.

Faut-il voir en eux le «grand-père», les «parents» et l'enfant (officieusement adopté)? En effet, Picquot appelle parfois Élise et Florent ses «enfants», et se qualifie souvent lui-même de «vieux». Ce dernier élément du support-signifiant Aurélien Picquot a sans doute pour signifié - tout comme son état de santé préoccupant - sa mort prochaine. Dans l'univers de l'après-texte, ce personnage français disparaîtra peut-être, mais seulement après avoir joué le rôle du parfait adjuvant et avoir fortement revêtu la fonction bremondienne d'influenceur auprès d'Élise et de Florent (qui aura assimilé certaines de ses valeurs, dont l'insistance sur la qualité en matière de cuisine). Tout se passe comme si le père français ancestral, disparu en 1760, était revenu pour donner un coup de main avant de redisparaître, ayant restauré le caractère français de la société-fille à laquelle il aura assuré d'ailleurs l'indépendance économique.

La double allégeance québécoise et française est au coeur de la signification de Maman-Paris Maman-la-France de Claude Jasmin. Le rapport que propose ce roman entre personnage français complexe et personnage québécois complexe relève, non plus de la cordialité qui marquait les rapports entre Tino Rossi et ses spectateurs québécois dans la Duchesse et le roturier, ni seulement du rapport père-enfant qui se tisse entre Aurélien Picquot et le couple Florent-Élise (bien que ce rapport se retrouve dans Maman-Paris Maman-la-France), mais bien d'une relation carrément fusionnelle.

Maman-Paris Maman-la-France abonde en personnages français solidaires des efforts du Québec et des Québécois de s'affirmer au Québec comme au Canada et sur le plan international. Plus d'un reprend à son compte le «Vive le Québec libre!» gaullien, d'autres déplorent l'abandon de 1760 ou encore le fait que Napoléon n'ait pas repris le Canada pour la France ${ }^{16}$. Ces personnages français

$16 \mathrm{Vu}$ les dimensions du présent travail, une nuance méthodologique s'impose ici en raison du fait que Maman-Paris Maman-Ia-France situe son intrigue en France, et que la grande majorité de ses personnages sont français (à l'inverse des autres romans). Dès lors, l'espace ne permet plus l'étude de personnages 
ont plus d'estime pour le parler québécois que n'en ont le narrateur et personnage principal québécois, Clément Jobin, et les autres personnages québécois. Pour Jobin, même les voyous parisiens parlent un français merveilleux pour nos oreilles écorchées de parlures québécistes (MPMF, p. 50). Il s'extasie sur la douceur de la parlure à Paris, qu'il qualifie de musique perpétuelle et de ravissement, tout en valorisant l'élocution française aux dépens de notre accent rocailleux et de cette mollesse dans nos dictions (MPMF, p. 21). L'enthousiasme que Jobin éprouve pour la phrase énoncée par un motard français (Dis donc mon vieux, je sais pas ce qu'elle a ma machine, mais elle gronde dans les virages!, MPMF, p. 21) et l'opposition que Jobin établit entre cette phrase et celle qu'aurait prononcée en pareille circonstance, selon lui, un motard québécois (Hostie d'calvaire, chum, mon bike buck dans les curves, tabarnak!, MPMF, p. 21), sous-entend qu'il voit en la variante hexagonale du français un instrument permettant de contrer l'envahissement anglophone dans la culture québécoise. Le responsable culturel de la Délégation générale du Québec à Paris avertit Rachel, conjointe de Clément et candidate à un prix de photographie francophone, $\mathrm{Ne}$ parlez pas trop, les Québécois n'ont rien à gagner dans ce domaine [...] (MPMF, p. 35). Ces personnages québécois vivent le langage des personnages français comme un modèle à suivre, attitude révélatrice de la même gêne qui, dans la Duchesse, noue la langue aux Québécois dès qu'ils constatent que Giscard d'Estaing est français (DR, p. 82). L'on n'observe, chez les personnages de Maman-Paris Maman-la-France, nulle trace de l'orgueil du langage québécois qui poussait Maryse à rejeter la norme française, jusqu'à ce qu'elle noie le génie de la langue française et se sente libre de choisir, indépendamment de toute contrainte extérieure, le français standard. Les Français deviennent, par le biais de leur langage, des influenceurs pour Clément et Rachel qui décident d'essayer constamment de mieux [s']exprimer (MPMF, p. 329-330). Et surtout, sur ce plan du langage comme sur d'autres, ils vivent explicitement et intensément les Français et Françaises comme adjuvants.

Le sentiment d'euphorie chez Clément et Rachel confirme l'adjuvance du personnage français complexe dans leur quête de bonheur, de dignité et de confiance. Quête d'identité aussi, et d'identité retrouvée: Clément a le sentiment d'être avec les Français parmi les siens, au sens et affectif, et biologique; il insiste sur les gènes qu'il partage avec eux (MPMF, p. 19,49).

Le Matou thématise fortement la vitalité d'Aurélien Picquot; de même, pour Clément, la vitalité constitue l'essence des Français, leur qualité prédominante, et il préfere les Québécois qui ont de la vitalité - ceux donc qui expriment

français individuels; heureusement, les personnages français de ce roman sont, avec leur culture et leur langue, autant de manifestations d'un phénomène textuel qui les englobe tous, et qui est le personnage français complexe tel que nous l'avons défini. L'essentiel, dans Maman-Paris Maman-la-France, sera la façon dont Clément et Rachel, héros québécois du roman, vivront ce personnage français complexe qui les entoure. 
l'essence du caractère français et sont ainsi les plus français, pour suivre sa logique (MPMF, p. 176-177). Il déplore que tant des nôtres so[ien]t comme endormis, apathiques (MPMF, p. 177) et se demande si la raison en est d'avoir cohabité de trop pres et trop longtemps avec les froids Anglo-Saxons, ou encore d'avoir été dépossédés de nos racines françaises depuis plus de deux cents ans (MPMF, p. 177). Par contre, il dit des Français que cette vitalité, Ce féroce goût de vivre [...] cet appétit de vie [...], cette vivacité dans les propos et les gestes font plaisir à voir, et il ajoute: N'est-ce pas cela, maman, exactement, être vivant vraiment? (MPMF, p. 177). Il semblerait bien que Jobin voie ici les Français et Françaises comme des adjuvants à un niveau profond, voire essentiel, pour luimême et potentiellement pour tous les Québécois, puisque ces Français(es) pourraient leur apporter un supplément de vitalité, voire de vie (N'est-ce pas cela, maman, exactement, être vivant vraiment?). L'image de l'adjuvance atteint ici son degré le plus fort dans notre corpus.

Comme l'indique le titre du roman, Jobin vit consciemment Paris et la France comme une mère, à la fois nouvelle et retrouvée (l'envahisseur britannique est traité de père fouettard et affairiste, MPMF, p. 288). Il se trompe peut-être en croyant que le personnage français complexe constitue uniquement une mère pour lui. Certains indices font penser que le personnage français complexe représente ici autant un père qu'une mère pour le personnage québécois. Par exemple, Rachel ne nomme que des hommes dans sa déclaration d'appartenance à la civilisation française:

Tous ces grands esprits sont notre héritage d̀ nous, les «parlant français» du monde [...]. Je suis fière d'être une Française d'Amérique, toutes ces gloires, regarde par là, Daudet, par ici, Delacroix et Beaumarchais, ce sont nos ancêtres à nous aussi, des Français comme nous! (MPMF, p. 333 , nous soulignons)

Voilà la fusion dont nous parlions: le personnage québécois ne voit plus le personnage français complexe comme l'autre - même adjuvant, voire parental - il est devenu lui-même français: Jobin termine le livre en déclarant: Je reviens chez moi, plus Français d'Amérique que jamais (MPMF, p. 344).

Gardons à l'esprit les observations que l'étude de ces quatre romans a permises, tout en nous penchant maintenant sur le Crime d'Ovide Plouffe. Ce roman se prête à un traitement indépendant. D'une part, il est le seul de notre corpus à comporter deux personnages français importants, mais de fonction signifiante très différente. Et surtout, si comme d'autres romans de notre corpus le Crime d'Ovide Plouffe implique la France et la culture française dans les significations du personnage français, il offre la particularité d'y impliquer fortement le problème précis des rapports entre écrivains français et québécois sur le champ (de bataille) du marché littéraire francophone.

À première vue, la problématique du personnage français dans le Crime d'Ovide Plouffe semble assez simple. D'un côté, il y aurait le Grenoblois 
Pacifique Berthet, partenaire commercial mais aussi opposant du héros québécois Ovide Plouffe. Ce Français, pour se venger d'une femme l'ayant humilié (Rita, épouse d'Ovide) et pour toucher cent mille dollars d'assurance, fait sauter en plein vol un avion transportant vingt-trois personnes. Ovide n'ayant pas pris le vol, Berthet essaie de le faire pendre pour le crime dont il devait être victime! De l'autre côté, opposée à ce monstre, il y aurait l'infiniment belle et très douce Parisienne, Marie Jourdan.

Plus complexes sont la présence et le fonctionnement signifiant réels des personnages français dans ce roman qui en fait en comporte beaucoup dont la plupart représentent un aspect de la culture française, le plus souvent la culture populaire (références fréquentes à Charles Trenet et à d'autres chanteurs et chanteuses français). Ces personnages sont presque toujours perçus positivement par les personnages québécois, comme des adjuvants dans leur quête d'un bonheur modeme, à l'instar de Tino Rossi dans la Duchesse et le roturier.

Représentant la grande culture musicale française (et sans doute, par métonymie, les autres arts aussi), Francis Poulenc aide à faire naître l'amitié entre Ovide et Marie. Enfin, Stendhal, comme auteur du Rouge et le noir, est souvent nommé par le roman qui lui assigne une fonction signifiante capitale.

Pacifique Berthet lui-même, malgré sa fonction actantielle monstrueuse, comporte sur le plan actoriel nombre de traits positifs: efficacité professionnelle (COP, p. 116), une excellente idée commerciale (la bijouterie), la force et la volonté de travailler de douze à quatorze heures par jour. Berthet a été exploité par l'oncle d'Ovide, Gédéon, fait qui rend d'autant plus estimable son travail comme adjuvant objectif d'Ovide. Et plus ardemment que quiconque, Berthet a cru au Canada: - Moi, Français de Grenoble, j'ai cru au Canada, j'ai cru aux défricheurs, aux aventuriers canadiens. Le malheur que vous savez m'a empêché de me joindre d eux (COP, p. 118). Ce malheur est une tuberculose coxalique déclenchée par une chute sur un trottoir glacé, bien métonymique du Canada qui s'avère ainsi cruellement injuste envers cet immigrant qui avait cru en son pays d'adoption! Le roman suscite donc délibérément chez le lecteur compassion et estime pour cette victime du sort, et souligne souvent son handicap et les qualités nécessaires pour le compenser: Que d'énergie, que de génie doit déployer un homme qui ne marche pas pour immobiliser un homme qui marche! (COP, p. 116, nous soulignons).

Le Crime d'Ovide Plouffe tend même à disculper Pacifique de son crime horrible en transférant le blâme sur Rita. Ayant attisé par ses flirts et adultères le désir de l'horloger presque privé de femmes par son infirmité, Rita refuse sa demande d'un simple baiser - qu'il fait presque pleurant - par une phrase cruelle qui transforme cette infirmité en véritable castration: - Jamais! Sale infirme! (COP, p. 202). Humiliation terrible, cette phrase tend à transférer une partie du blâme, pour l'attentat contre l'avion, de Pacifique à Rita. 
Est-ce dire que, n'eût été Rita, Pacifique aurait été pour Ovide l'adjuvant bon père qu'est Picquot pour Florent dans le Matou, et qu'il n'est pas opposant? Certes non; mais les procédés par lesquels ce roman tend à valoriser Berthet et à le disculper partiellement de son premier crime (l'attentat), nous avertissent qu'il faut chercher ailleurs et plus loin - dans les chapitres consacrés au deuxième méfait de Berthet (sa tentative de faire pendre Ovide pour l'attentat) - le lieu véritable de l'affrontement entre personnages québécois et français dans ce roman. Pacifique Berthet est-il faux adjuvant, traître déguisé? Bien sûr; mais pour comprendre la signification de ce personnage, il faudra tenir compte aussi bien d'une autre double identité que le roman lui assigne avec insistance que de la lutte de Berthet avec un tout autre Québécois qu'Ovide - Denis Boucher.

Mais d'abord, il faut se pencher sur le personnage français de Marie Jourdan, ce qui nous amènera à considérer aussi ce Paris et cette France qui lui sont métonymiquement liés. Marie Jourdan est indiscutablement le symbole d'une certaine France et d'une certaine culture française.

Elle représente d'abord, l'apogée du beau: sa beauté physique, présentée comme parfaite, absolue, est soulignée à presque chaque mention du personnage. Le caractère absolu de sa beaute signale que celle-ci n'est pas qu'un trait physique éveilleur du désir masculin (comme l'est la beauté de Rita), mais l'incarnation d'un idéal d'ordre esthétique. La grande douceur du personnage conjugue ses effets signifiants à ceux de sa beauté: ensemble, ces deux traits font de Marie le symbole d'une certaine France mythique, la belle et douce France de Du Bellay. L'aura d'esthétisme qui entoure ce personnage signifie que Marie Jourdan représente aussi une attitude envers la vie, une attitude privilégiant les valeurs esthétiques (cf. A. Picquot); elle s'en rend compte après avoir perdu son emploi à cause de sa beauté même:

Qu'allait-elle devenir? [...] On la pénalisait parce qu'elle était trop jolie, parce qu'elle soignait ses manières, parce qu'elle avait une certaine éducation, parce que son accent était parisien et qu'elle ne se permettait pas de vulgarité. Pourtant on lui avait tellement dit qu'au Québec elle trouverait le paradis! Quelle société était-ce, où l'on s'acharnait contre les défenseurs d'une certaine façon de vivre, comme si on voulait les faire entrer dans le rang, vers le bas? (COP, p. 263, nous soulignons)

Le goût de Marie pour la musique de Francis Poulenc ne fait que confirmer sa fonction signifiante actorielle: représenter une certaine culture française caractérisée avant tout par la position suprême qu'elle accorde aux valeurs esthétiques.

Ovide Plouffe est tellement ému de voir entrer dans le magasin de disques qui l'emploie une telle incamation de la culture française qu'il s'évanouit! (COP, p. 48). L'acte qu'accomplit alors Marie est significatif, car s'y conformera la conception que se fera Ovide de la fonction actantielle de Marie par rapport à lui: Marie humectait doucement le visage d'Ovide (COP, p. 48). Marie essaie aussi d'aider Ovide, et celui-ci recherchera toujours en elle un adjuvant. Ce sera vrai 
même lorsque Ovide lui offrira d'être sa secrétaire: ce qu'il recherchera alors sera une compagnie apte à le consoler (au moral) de la trahison de Rita: Ne nous laissons pas, Marie, aidons-nous l'un l'autre, voulez-vous? fit-il en l'étreignant comme un noye (COP, p. 267, nous soulignons). La fascination d'Ovide pour Marie la Française (comme le roman l'identifie dès leur première rencontre (COP, p. 46), et son insistance à voir en elle un adjuvant plutôt que la princesse-àconquérir, l'«objet» en terminologie actantielle, sont fortement liées à sa perception de Marie comme l'incarnation parfaite de l'essence même de la France - c'est-à-dire à sa fascination pour la France et la grande culture française. Exalté, il déclare à Marie: Toute ma vie, j'ai lu, j'ai vécu dans la hantise de la Ville-lumière (COP, p. 293). Il l'avoue aux policiers: Depuis mon enfance, je rêve de Paris, des Champs-Elysées, de Notre-Dame, de la culture française, de l'opéra (COP, p. 373). Cette hantise conduit à une rêverie de la fusion: lors de la fête du 14 juillet au consulat de France, dans l'ivresse déclenchée par l'accent parisien des conversations fusant autour de lui, Ovide pense que:

Au fond, il était un Français déraciné depuis trois cents ans, perdu dans ce Québec sibérien mais qui, aux effluves de ses lointaines origines, humait avec délices sa véritable identité. Oui, un Français, et il le redisait à chacun avec une fierté qui suggérait presque le reniement de son statut de Canadien. (COP, p. 344)

Il est clair que l'ensemble France-culture française, qu'Ovide conçoit comme un être féminin (d'ailleurs incarné en la personne de Marie Jourdan), représente pour lui une instance maternelle. Ce fait peut aider à expliquer pourquoi il recherche en Marie un adjuvant, une mère, cet ange pur et radieux qu'évoque son prénom, au lieu de la voir comme une femme à conquérir. Attitude et comportements qui ne surprennent pas de la part d'Ovide, catholique, timide, idéaliste, velléitaire.

D'autres Québécois dans ce roman ont envers Marie une attitude et un comportement différents. Ils voient en elle l'objet de leur quête, une femme à conquérir. Tit-Mé et Guillaume manifestent à Marie leur désir et Guillaume, guide de chasse et de pêche, sera recompensé de sa hardiesse physique envers Marie par un après-midi d'amour passionné. Mais un seul.

Celui à qui serait promise la conquête définitive de Marie-la-Française/Mariela-France, c'est un Québécois d'une autre trempe, s'étant trempé justement dans la grande Amérique, comme il dit (COP, p. 409), journaliste au Time, à New York: Denis Boucher.

Les chapitres qui suivent l'arrestation d'Ovide (p. 356-500) réduisent celui-ci à un rôle secondaire; il n'est plus sujet, mais objet assez pitoyable qu'il faut sauver. Se dessine ainsi une grande lutte finale qui promeut Denis au rang de héros et nous permettra de mieux cerner la problématique du personnage français dans ce roman, puisque le grand adversaire du sujet-héros québécois Denis Boucher sera Pacifique Berthet. Il est temps donc de retoumer à ce personnage à 
double identité pour en dégager la véritable signification ainsi que celle de la lutte entre Berthet et Boucher, Français et Québécois.

Ovide, dès l'époque de sa première rencontre avec Berthet, nous livrait la signification veritable de celui-ci en se rappelant pourquoi le nom de «Berthet», associé à Grenoble, lui disait quelque chose:

- Mais oui, je l'ai. [...] Eurêka! Antoine Berthet, ce garçon dont le crime relaté dans la GAZETTE DES TRIBUNAUX a inspiré Stendhal dans la création de son roman Le Rouge et le Noir, dont l'action se situe à Grenoble. (COP, p. 64)

Or, cette identification de Pacifique Berthet à Antoine Berthet, au Rouge et le Noir et à Stendhal, sera rappelée maintes fois au cours du roman avec une insistance fort significative. L'insistance avec laquelle le texte répète cette série de signes, cette équation, lui confere une incontournable fonction signifiante: celle de mettre en équivalence Pacifique Berthet et l'ensemble constitué par le romancier et le roman français, ensemble que représentent métonymiquement Stendhal et son roman. D'autant plus que Berthet, par ses mensonges, se mue en créateur de fictions. La fonction signifiante fondamentale de Pacifique Berthet consiste donc d'une part, à être le symbole même du roman et des romanciers français, et d'autre part, à être opposant.

Pacifique perd, Boucher gagne. Que signifie ce triomphe? Dans la mesure où Berthet symbolise les romanciers français et leurs romans, et où Boucher mise sur l'écriture pour se réaliser, cette lutte semble opposer deux groupes nationaux d'écrivains, français et québécois, et peut-être aussi deux traditions romanesques. Nous objecterait-on que nous cherchons à tort à comparer l'incomparable, l'écriture romanesque et ses praticiens français d'une part, et l'écriture journalistique (à l'américaine) de Denis Boucher, d'autre part? Mais c'est précisément là ce que fait ce roman d'un ancien journaliste, ancien directeur de la Presse. Le Crime d'Ovide Plouffe signale que Denis, tout comme Ovide, avaient beaucoup admiré Ces grands noms, Stendhal, Berthet, puis celui de Julien Sorel, à qui Ovide et lui-même s'étaient identifiés jadis [...] (COP, p. 419). Voilà Denis, journaliste, rapproché professionnellement de Stendhal, romancier, et de l'activité romanesque. D'ailleurs, Le romancier soudain possédé par un grand sujet n'est pas plus exalté que Denis Boucher le fut quand il mit les pieds dans les rues de la paroisse [...] (COP, p. 417) - c'est-à-dire, au moment où il commence son enquête et par là même son projet d'écriture.

Lui-même romancier et journaliste, Lemelin, en mettant sur un même plan écritures romanesque et journalistique ainsi que leurs praticiens, ne fait qu'affirmer sa vision des parentés entre les deux types d'écriture et donc entre les deux professions: il implique que tout roman est dans une certaine mesure écriture de vérité, et que tout reportage comporte un travail de fiction, ne serait-ce que celui du choix et de la disposition de ses éléments. 
Mais si ce roman rapproche romanciers et journalistes, c'est pour mieux rendre compte d'une opposition qui les dresserait les uns contre les autres: Lemelin semble bien ici se pencher sur le champ de bataille où s'opposeraient écrivains français et québécois, à savoir le marché littéraire (et plus précisément romanesque) francophone. Il est évident qu'avec ce roman Lemelin ne vise pas que le marché québécois, d'où la grande fréquence et limportance des personnages français et des références à la France et à la culture française dans le Crime d'Ovide Plouffe, d'où même l'emploi du système métrique: au minimum, le marché français s'ajoute au québécois dans la visée commerciale de l'auteur 17 . Que ce roman, à un certain niveau de signification, oppose écrivains québécois et français, voilà qui fait ressortir sa fonction conative, (celle par laquelle, selon les linguistes, le texte vise à agir sur ses destinataires 18 ), et aussi sans doute, une opposition entre deux traditions romanesques. Lemelin, qui manifestement par la victoire de Boucher prend position en faveur de la réussite des écrivains québécois dans cette compétition, semble bien s'adresser avant tout à une partie précise de son lectorat - ses collègues romanciers québécois - afin de les convaincre de miser, non pas sur le modèle français (ce qui pourrait les inciter à imiter la tradition romanesque française), mais bien plutôt sur leur avantage spécifique leur américanité. Cette américanité leur permettrait de mieux concurrencer les romanciers français sur le marché francophone en offrant à celui-ci ce que ne peuvent lui offrir ceux-là: des romans «américains» en français, formule concourant depuis quelque temps à la réussite du roman québécois en France, Jacques Allard l'a bien démontré19.

Inquiétant toutefois est le prix dont Denis paie la victoire de son écriture: exilé aux États-Unis, il écrit non seulement à l'américaine, mais en américain. Le texte de Lemelin signifierait-il donc aux romanciers québécois que, pour réussir, ils doivent écrire en anglais? Cela nous semble douteux, car il y aurait alors contradiction entre ce message et le texte (Ecrit en français) qui le ferait exister. Le personnage de Boucher-Lemelin se rapprocherait plutôt d'un certain type de Québécois contemporains prêt à internationaliser leurs expériences, leur itinéraire, et l'espace de leur quête, et dont les symboles dans la mythologie québécoise contemporaine sont des sociétés comme Lavalin et Bombardier.

17 Nous avons pu constater à Marseille en 1982 (année de la publication du Crime d'Ovide Plouffe) l'intérêt que R. Lemelin porte au marché français: lors d'une pré-projection du film les Plouffe de Gilles Carle basé sur un de ses romans, Lemelin, devant une grande salle de cinéma emplie de spectateurs invités, a prononcé un discours dans lequel il a fait de son mieux pour vendre le film, et lui-même en tant qu'auteur, au public marseillais en insistant notamment sur ses liens avec des écrivains provençaux, dont surtout Marcel Pagnol, argument de vente particulièrement efficace à Marseille.

18 Roman Jakobson, «Linguistique et poétique», Essais de linguistique générale, Paris, Minuit, 1963, p. 209-248.

19 Jacques Allard, «Des branches et des arbres», Francofonia, no 10, Primavera 1986, p. 12-23. 
Preuve finale que la lutte entre Denis Boucher (représentant de Lemelin et des écrivains québécois) et Pacifique Berthet-Stendhal a pour enjeu la place de l'écrivain québécois, c'est le fait que, dans l'«Épilogue», Boucher reçoive une double récompense: promu et mutê à Paris, il y est en voie de conquérir le coeur et le corps de Marie Jourdan, symbole de la France et de la culture française. Boucher, au lieu de voir en Marie une mère et un adjuvant, comme la voyait Ovide, la voit comme une femme à séduire, à conquérir, tout simplement. Le roman implique que la conquête définitive de Marie ne pouvait échoir ni à Ovide, représentant du Canada français catholique, ni même au viril Guillaume, symbole archaïsant peut-être, de par son mode de vie, voire son habillement, des aventuriers et coureurs des bois de la Nouvelle-France, mais devait couronner un Québécois moderne, Denis Boucher, qui se définit avant tout par son américanité. La conquête de Marie par Denis constitue une réfutation non seulement de l'attitude d'Ovide qui voit en la France et sa culture une mère, mais aussi, dans une certaine mesure, de celle du héros de Maman-Paris Maman-la-France. La conquête de Marie-la-Française/Marie-la-France par Denis constitue fondamentalement une réfutation de l'attitude de tous ceux qui, au Québec, auraient été tentés d'admirer béatement et d'imiter servilement la culture française. C'est aussi un appel aux écrivains québécois pour qu'ils s'affirment sur le marché littéraire francophone et recourent, face à la concurrence des Français, à leur avantage spécifique, leur américanité.

Ainsi, le Crime d'Ovide Plouffe, tout à la fois se rapproche-t-il et se démarque-t-il des autres romans de notre corpus. Tout en confiant à son tour au personnage français la fonction de symboliser la France et la culture française, il élargit le champ et l'enjeu des rapports entre personnages français et québécois pour englober l'espace français, voire l'univers culturel francophone. Cet élargissement du champ géographique s'accompagne d'une localisation précise du champ d'activité culturelle concernée: le domaine littéraire.

Enfin, dans le Crime d'Ovide Plouffe, la lutte de l'écrivain québécois contre son homologue français prend l'allure d'une révolte contre le Père, comme l'indiquent la différence d'âge entre Pacifique et Denis, et surtout le fait que ce soit Stendhal et la grande tradition romanesque française qu'il symbolise qui représentent les romanciers français, plutôt qu'un contemporain de Denis.

Pour conclure, sur le plan méthodologique, notre analyse a privilégié au départ le volet actantiel du personnage, la répartition entre personnages: opposants et adjuvants nous a semblé fondamentale pour établir la signification du personnage français dans notre corpus, signification qui a paru d'emblee relative - c'est-à-dire découler de la relation entre personnages français et québécois. En outre, de nombreux traits des divers personnages français, en tant qu'acteurs et plus précisément comme supports-signifiants, se sont révélés porteurs de signifiés importants pour la signification d'ensemble du personnage français - y compris sa pleine signification actantielle. Ce qui confirme l'interdépendance des diverses composantes du personnage romanesque proposées dans notre définition. 
Le personnage français complexe assume des fonctions signifiantes importantes, et parfois fondamentales, dans les cinq romans étudiés. Le personnage français humain est le plus souvent masculin dans notre corpus (comme l'est la langue française quand elle se matérialise sous la forme humanoïde du génie de la langue française dans Maryse). Associé à d'autres, ce fait textuel permet d'avancer l'hypothèse selon laquelle la fonction signifiante principale du personnage français complexe dans notre corpus serait de constituer une image paternelle, comme on l'a vu dans le Matou et Maman-Paris Mamanla-France, romans dans lesquels le personnage français complexe joue largement un rôle patemel positif, un rôle de «bon père» 20 . Cette fonction de limage patemelle demeure même lorsque le personnage français devient un «mauvais père», comme dans Maryse et le Crime d'Ovide Plouffe. Le personnage français est perçu négativement par les personnages québécois surtout lorsqu'il veut s'arroger toute autorité et tout pouvoir sur la langue française, la langue maternelle, la langue de la mère, interdisant ainsi toute emprise québécoise sur cette langue maternelle ou (dans le cas du Crime d'Ovide Plouffe) ses manifestations littéraires. Mais la fonction de figure paternelle positive atteint un degré et une intensité inégalés dans le Matou et dans Maman-Paris Maman-laFrance: les remarques suivantes porteront surtout sur ces deux «cas limites» de la figure paternelle positive.

Que ce personnage français puisse constituer avant tout une image paternelle, contre laquelle le personnage québécois se révolte, ou dont il cherche à retrouver la présence, l'approbation, voire la fusion, c'est ce que suggérerait un rapprochement de notre corpus avec l'article de Claude Galarneau, «la Légende napoléonnienne au Québec». Galarneau, à propos des conséquences de la Conquête sur les Canadiens français, affirme que: les masses populaires, chrétiennes et monarchistes [...] avaient perdu leur mère patrie sans comprendre, mais étaient restées très attachées au roi de France. [...J Et soudain la Révolution, décapitant Louis XVI, les prive de leur père 21.

20 Nous sommes redevable à André Vanasse de nous avoir fait observer, suite à notre communication au colloque ALCQ 1986 (voir note 1 ci-dessus), que nos remarques sur la fonction paternelle du personnage français et selon lesquelles celui-ci est mal perçu surtout lorsqu'il veut s'arroger un pouvoir absolu et exclusif sur la langue maternelle, pouvaient s'enrichir d'une analyse en termes de «bon père» et de «mauvais père». A. Vanasse a fait remarquer que dans le Matou le vrai père de Florent a un rôle peu important; qu'il est, en quelque sorte, évacué du récit. Nous ajouterons que le peu de succès qu'a ce père dans ses tentatives d'aider Florent tend à l'affecter d'un coefficient d'impuissance assez significatif; le vrai père est d'une présence tout aussi chancelante dans les autres romans de notre corpus. Florent préfère recevoir l'aide du père «adoptif» qu'est le Français Aurélien Picquot, plutôt que de son vrai père québécois.

21 Claude Galarneau, op. cit., p. 167. 
Toujours selon Galameau, ce sevrage expliquerait l'accueil fait au Québec du mythe napoléonien, un Napoléon héros, homme du destin et substitut paternel. $Y$ aurait-il un rapport entre cette attitude et le fait qu'Aurélien Picquot, pour sensible et bon qu'il soit, est souvent décrit comme autoritaire et comparé explicitement tantôt à Napoléon (Mat., p. 514), tantôt à un général préparant la bataille (Mat., p. 526)?

Yves Beauchemin a commencé son premier roman, l'Enfirouapé (1974), par l'évocation du capitaine d'artillerie français Rodrigue Ferland de la Barre, arrivé en 1665 à Québec. Il fut à l'origine d'une longue lignée de Ferland de la Barre que l'arrivée des Anglais réduisit au simple nom de Ferland et, à terme, à l'état de chômeurs 22. Le batailleur Picquot ne serait-il pas un avatar de ce père ancestral, Ferland de la Barre, revenu aider sa descendance québécoise à redresser la barre avant de les quitter à nouveau, mais imprégnée de lui au point de s'identifier à ce père ancestral, comme dans Maman-Paris Maman-la-France23?

«Redresser la barre» est une fonction qui semble prendre deux formes ici. D'une part, renforcer le caractère français de la société québécoise (c'est la principale fonction assignée au personnage français dans Maman-Paris Mamanla-France, mais elle n'est pas absente des fonctions d'Aurélien Picquot qui aide un Québécois francophone contre des adversaires le plus souvent non francophones); d'autre part, aider les Québécois à accéder à une position de pouvoir et d'indépendance économiques (c'est la principale fonction d'Aurélien Picquot). Antoine Sirois, étudiant l'étranger de race et d'ethnie dans le roman québécois de 1918 à 1974, remarque que les Français y sont dans l'ensemble décrits de façon positive (malgré l'abandon de 1760 et la Révolution française), en raison du fait que les romanciers québécois de son corpus voyaient ces personnages dans une optique principalement culturelle, liée à l'effort de défendre la langue. Sirois va jusqu'à écrire que Beaucoup tentent de retourner aux sources françaises 24 , phrase appliquable à l'attitude de Clément et de Rachel dans Maman-Paris Maman-laFrance.

L'importance de la fonction économique chez le personnage d'Aurélien Picquot marque donc une évolution assez nette par rapport à ce qu'a observé $\mathrm{A}$. Sirois dans un corpus antérieur (mais sa remarque à caractère général tolère quelques exceptions: ainsi, notre lecture du héros du Français de Damase Potvin, paru en 1925 et auquel nous consacrons actuellement une étude, nous a convaincu que ce personnage revêt à la fois d'importantes fonctions culturelles et économiques). Si la fonction générale consistant à aider le personnage québécois à «redresser la barre» peut prendre une forme tantôt culturelle, tantôt économique (et les deux peuvent avoir des résonances d'ordre politique, bien sûr), ces deux

22 Yves Beauchemin, l'Enfirouapé, Montréal, Stanké, coll. «10/10», no 72, p. 5.

23 Voir à ce sujet nos remarques sur A. Picquot, ci-dessus p. 92-93.

24 Antoine Sirois, op. cit., p. 198. 
formes restent compatibles avec la fonction paternelle que semble revêtir le personnage français dans l'ensemble de notre corpus.

Le Crime d'Ovide Plouffe, qui thématise l'opposition entre Québécois et Français, place cette opposition sur le plan des rapports entre écrivains, mais ces rapports en sont précisément de concurrence. Dès lors, la problématique des rapports entre Français et Québécois comporte encore une fois une dimension économique importante, mais ces rapports n'en sont plus d'entraide, mais de compétition. Dans la mesure où Pacifique Berthet-Stendhal et donc l'écrivain français figurent une instance paternelle, ils apparaissent comme le Père qui ne veut pas céder au fils les moyens de production économique. Mais en même temps l'instance maternelle Marie/la France devient objet de quête (et de conquête) pour l'écrivain québécois, qui y trouvera donc l'épanouissement économique que permettra le marché littéraire français.

Nous espérons l'avoir démontré dans le cadre de notre corpus: constituer une image patemelle semble être la fonction signifiante fondamentale du personnage français dans le roman québécois contemporain. Cette hypothèse devra subir l'épreuve d'un corpus plus étendu. Indépendamment de cette hypothèse toutefois, le personnage français, tant humain que complexe, comme acteur et comme actant, opposant ou adjuvant, a confirmé sa pertinence et son intérêt précieux pour une meilleure compréhension du corpus romanesque québécois, voire des rapports culturels, affectifs, intellectuels et économiques entre les deux peuples.

\section{Bibliographie}

ABOUTEBOUL, Albert-Victor, le Personnage juif dans le roman canadien-français du vingtlème slècle, mémoire de M.A., McGill University, 1971.

HATHORN, Ramon, le Monde anglo-saxon dans le roman canadien-françals, thèse de doctorat, Université d'Ottawa, 1974; «Angles on Saxons: A Study of the Anglo-Saxon in Quebec Fiction», Journal of Canadian Fiction no. 25-26, 1979, p. 264-279; «Soldats, patrons et femmes fatales: figures de l'«Anglais» dans le roman québécois des XIXe et XXe siècles», Voix \& images, VI, 1 , aut. 1980 , p. $97-115$.

ROUSSAN, Wanda de, la Signification du personnage de l'étrangère dans le roman québécois contemporain, mémoire de maitrise, Université de Montréal, 1973.

SHEK, Ben-Z., «l'Image des Juifs dans le roman québécois», dans P. Anctil et G. Caldwell (dir.), Juifs et réalités juives au Québec, Québec, Institut québécois de recherche sur la culture, 1984, p. 255-286

SIROIS, Antoine, «l'Étranger de race et d'ethnie dans le roman québécois», dans F. Dumont et $Y$. Martin (dir.), Imaginaire social et représentations collectives, Mélanges offerts à Jean-Charles Falardeau, Québec, Presses de l'Université Laval, 1982, p. 187-204. 
TEBOUL, Victor, Mythe et images du juif au Québec, Montréal, Québec, Editions de Lagrave, 1977, 235 p. Voir aussi de Teboul: «Antisémitisme: mythe et images du Juif au Québec (essai d'analyse)», Voix \& images du pays IV, avril 1971 , p. $53-81$

VANASSE, André, «la Rupture définitive. La notion d'étranger dans la littérature canadienne-française», l'Action nationale, mars 1966, p. 606-611.



